\title{
EFFECT OF DAMAGES ON CRACK DEVELOPMENT IN COATING ON ELASTIC FOUNDATION
}

\author{
Vagif M. Mirsalimov \\ Institute of Mathematics and Mechanics of NAS of Azerbaijan, Baku, Azerbaijan Republic \\ e-mail: vagif.mirsalimov@imm.az
}

Shahin G. HASANov

Azerbaijan Technical University, Baku, Azerbaijan Republic; e-mail: iske@mail.ru

\begin{abstract}
A fracture mechanics problem for a coating linked to the basis made of another elastic material is considered. It is assumed that in the vicinity of technological defect (crack) in the process of loading in the coating, there will arise prefracture zones (damages) that are modeled as the areas of weakened interparticle bonds of the material. In the prefracture zones (interlayers of the overstressed material), the coating material is deformed beyond the limit of elasticity. It is considered that during loading in the cross section of the coating in the vicinity of the crack, there is an arbitrary number of rectilinear prefracture zones. The condition determining the limiting value of the external load at which the crack growth happens is obtained.
\end{abstract}

Keywords: coating, elastic foundation, crack with plastic end zones, prefracture zones

\section{Introduction}

Analysis of the present state of coatings revealed that the coating materials have crack-visible discontinuities. In the cross sections of the coating, there arise transition zones where physico-mechanical features of the material differ from the features of the basic coating. The indicated damages in the coating cross section may have both natural origin (lamination, inclusions, pores) or be caused by technological processes. In spite of importance of the enumerated factors on coating strength, up today, these issues have not found due consideration in calculation methods. Development of calculation models of investigation of damage of a coating on an elastic foundation is a very urgent problem. A problem on interactions of damages on crack growth (Mirsalimov and Rustamov, 2012a,b) is an important problem of strength theory. Wide literature, e.g. Kulchytsky-Zhyhailo and Rogowski (2007), Haj-Ali (2009), Tukashev and Adilhanova (2010), Ameri et al. (2011), Hasanov (2010, 2013), Hasanov and Mirsalimov (2014) and others has been devoted to investigation of the stress strain state and fracture of the coating on an elastic foundation.

\section{Formulation of problem}

Consider with respect to Cartesian coordinates $x, y$ a double-layer body consisting of a coating of thickness $h$ with the elastic characteristics $G_{1}$ (shear modulus) and $\mu_{1}$ (Poisson ratio) linked with an elastic half-plane with characteristics $G_{2}$ and $\mu_{2}$ (Fig. 1).

Consider a fracture mechanics problem for a double layer body when the normal load $P$ is applied to the external surface of the coating. The remaining part of the coating is not loaded. It is accepted that the coating material has a crack with end plastic zones (Leonov-Panasyuk-Dugdale 


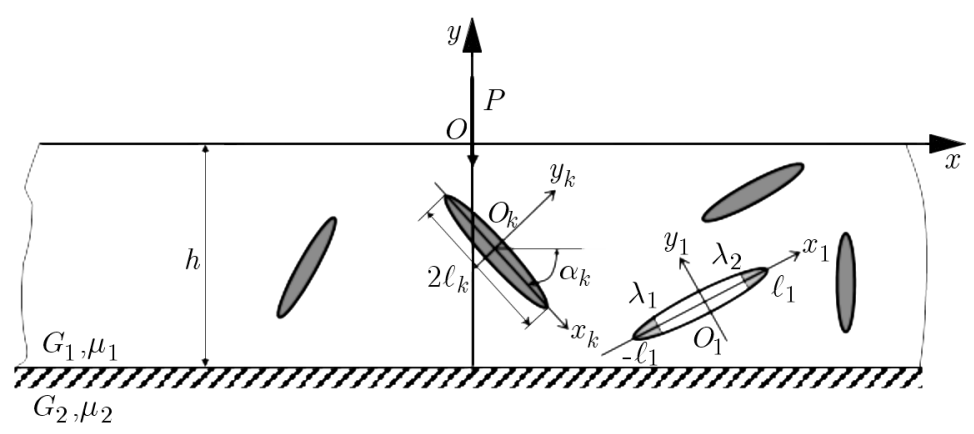

Fig. 1. A scheme of the problem of interaction of prefracture zones and cracks with end zones in the coating

crack model), see Leonov and Panasyuk (1959), Dugdale (1960). It is assumed that in the coating material in the vicinity of the crack, after repeated loading, there appear damages (prefracture zones) that are modeled as areas of interparticle bonds of the material. At the loading with external loading in the interlayer of the overstressed material a plastic flow is formed. Let, for definiteness, the power loadings change so that plastic deformation is realized in the area of weakened interparicle bonds of the material. Interaction of the faces of prefracture zones is modeled by the lines of plastic flow between them (degenerated plastic deformation zones). Under constant stress the sizes of plastic flow zones (prefracture zones) depend on the form of the material. General tendency to formation of areas with a broken structure of the material at early stages of fracture in the form of narrow layers occupying slight volume of the body compared with its elastic zone (Panasyuk, 1991; Mirsalimov, 1987; Rusinko and Rusinko, 2011) is known well from practice. The sizes of prefracture zones at the end plastic deformation zones at the crack tips are unknown beforehand and should be defined. Interaction of the prefracture zones in the vicinity of technological defect (crack) may reduce to the loss of crack stability, appearance of new cracks. It is assumed that the prefracture zones are oriented in the direction of action of maximal tensile stresses appearing in the coating.

Since the indicated zones are small compared with the remaining elastic part of the coating, one can mentally remove them changing it by cuttings whose surfaces interact between themselves by some law corresponding to the action of the removed material. In the coating cross section the crack with end zones is of length $2 l_{1}$ along the axis $x_{1}$. Let in the coating in the vicinity of the crack there will be $(N-1)$ prefracture zones of length $2 l_{k}(k=1,2, \ldots, N)$ (Fig. 1). In the centers of prefracture zones and the crack with end zones, we locate the origin of a local system of coordinates $x_{k} O_{k} y_{k}$ whose axes $x_{k}$ coincide with the prefracture zones and the crack, and make the angles $a_{k}$ with the axis $x$ (Fig. 1).

Under the action of the external power load $P$ on the coating surface in bonds connecting the prefracture zone faces and the cracks in end zones, there will arise normal $q_{y_{k}}=\sigma_{S}$ and tangential $q_{x_{k} y_{k}}=\tau_{S}$ stresses $(k=1,2, \ldots, N)$, where $\sigma_{S}$ is the yield point of the coating material for tension; $\tau_{S}$ is the yield point of the material for shear.

The boundary conditions of the problem are written in the form (the upper index 1 corresponds to the coating, the upper index 2 to the half-plane):

— for $y=0$

$$
\sigma_{y}^{(1)}=-P \delta(x) \quad \tau_{x y}^{(1)}=0
$$

- for $y=-h$

$$
u^{(1)}+\mathrm{i} v^{(1)}=u^{(2)}+\mathrm{i} v^{(2)} \quad \sigma_{y}^{(1)}+\mathrm{i} \tau_{x y}^{(1)}=\sigma_{y}^{(2)}+\mathrm{i} \tau_{x y}^{(2)}
$$

- for $y_{1}=0$

$$
\lambda_{11}<x<\lambda_{21} \quad \sigma_{y}^{(1)}=0 \quad \tau_{x y}^{(1)}=0
$$


- for $y_{1}=0,-\ell_{1} \leqslant x_{1} \leqslant \lambda_{11}$ and $\lambda_{21} \leqslant x_{1} \leqslant \ell_{1}$

$$
\sigma_{y_{1}}^{(1)}=\sigma_{s} \quad \tau_{x_{1} y_{1}}^{(1)}=\tau_{s}
$$

and

$$
\sigma_{y_{k}}^{(1)}=\sigma_{s} \quad \tau_{x_{k} y_{k}}^{(1)}=\tau_{s} \quad \text { on } \quad L_{k} \quad(k=1,2, \ldots, N)
$$

where $L_{k}$ are the faces of the $k$-th prefracture zone; $\delta(x)$ is Dirac's impulse function, $\sigma_{x}, \sigma_{y}, \tau_{x y}$ are stress tensor components; $u, v$ are displacement vector components: as $y \rightarrow-\infty$ the displacements and stresses disappear.

\section{The method of the boundary-value problem solution}

For the solution of the problem under consideration we use the superposition principle. Then we can represent the state of a double-layer body in the form of the sum of two stress-strain states:

1) adhesive connection of materials without a crack and prefracture zones under the action of the external normal load $P$ on the external surface of the coating;

2) stress-strain state of a coating with a crack and prefracture zones on the faces of which the stresses equal in value and opposite in sign, defined by the first stress-strain state for $y_{1}=0$ and on $L_{k}$ are additionally applied.

The boundary conditions for the first stress state are of the form (2.1)-(2.2).

For the solution of boundary value problem (2.1), (2.2), we use Papkovich-Neiber's four functions $F_{n}^{m}(x, y)(n, m=1,2)$. Two of them are for the coating (upper index 1) and two for the half-plane (upper index 2).

The stresses and strains are expressed by the Parkovich-Neiber function by the known formulae (Uflyand, 1967)

$$
\begin{aligned}
& \frac{\sigma_{y}^{(m)}}{2 G_{m}}=2\left(1-\mu_{m}\right) \frac{\partial F_{2}^{m}}{\partial y}-\frac{\partial^{2} F_{1}^{m}}{\partial y^{2}}-y \frac{\partial^{2} F_{2}^{m}}{\partial y^{2}} \\
& \frac{\tau_{x y}^{(m)}}{2 G_{m}}=\frac{\partial}{\partial x}\left[\left(1-2 \mu_{m}\right) F_{2}^{m}-\frac{\partial F_{1}^{m}}{\partial y}-y \frac{\partial F_{2}^{m}}{\partial y}\right] \\
& u^{(m)}=-\frac{\partial F_{1}^{m}}{\partial x}-y \frac{\partial F_{2}^{m}}{\partial x} \quad v^{(m)}=\left(3-4 \mu_{m}\right) F_{2}^{m}-\frac{\partial F_{1}^{m}}{\partial y}-y \frac{\partial F_{2}^{m}}{\partial y}
\end{aligned}
$$

Taking into account the symmetry of the problem in $x$, we use the Fourier cos-transformation. Accept that

$$
\begin{aligned}
& F_{1}^{1}=\int_{0}^{\infty}(A \sinh \alpha y+B \cosh \alpha y) \cos \alpha x d \alpha \\
& F_{2}^{1}=\int_{0}^{\infty}(C \sinh \alpha y+D \cosh \alpha y) \alpha \cos \alpha x d \alpha \\
& F_{1}^{2}=\int_{0}^{\infty} E \mathrm{e}^{\alpha y} \cos \alpha x d \alpha \quad F_{2}^{2}=\int_{0}^{\infty} F \mathrm{e}^{\alpha y} \alpha \cos \alpha x d \alpha
\end{aligned}
$$


Satisfying by functions (3.1), (3.2) boundary conditions (2.1), (2.2), we get a system of six linear algebraic equations with respect to six unknown functions $A(\alpha), B(\alpha), C(\alpha), D(\alpha), E(\alpha)$, $F(\alpha)$

$$
\begin{aligned}
& 2\left(1-\mu_{1}\right)(C \cosh \alpha h+D \sinh \alpha h)-A \sinh \alpha h-B \cosh \alpha h \\
& \quad-\alpha h(C \sinh \alpha h+D \cosh \alpha h)=-\frac{P}{2 \pi G_{1} \alpha^{2}} \\
& \left(1-2 \mu_{1}\right)(C \sinh \alpha h+D \cosh \alpha h)-A \cosh \alpha h-B \sinh \alpha h \\
& \quad-\alpha h(C \cosh \alpha h+D \sinh \alpha h)=0 \\
& B=E \quad\left(3-4 \mu_{1}\right) D-A=\left(3-4 \mu_{2}\right) F-E \\
& G_{1}\left[2\left(1-\mu_{1}\right) C-B\right]=G_{2}\left[2\left(1-\mu_{2}\right) F-E\right] \\
& G_{1}\left[\left(1-2 \mu_{1}\right) D-A\right]=G_{2}\left[\left(1-2 \mu_{2}\right) F-E\right]
\end{aligned}
$$

Solving algebraic system of equations (3.3) by the method of successive exclusion of unknowns, we find the coefficients $A(\alpha), B(\alpha), C(\alpha), D(\alpha), E(\alpha), F(\alpha)$

$$
\begin{aligned}
& D=\frac{\Delta_{1}}{\Delta} \quad F=\frac{\Delta_{2}}{\Delta} \quad E=B \\
& A=\frac{1}{a_{11}}\left(B_{1}-a_{12} B-a_{13} C-a_{14} D-a_{15} F\right) \\
& B=\frac{1}{c_{11}}\left(B_{2}-\frac{a_{21}}{a_{11}} B_{1}-c_{12} C-c_{13} D-c_{14} F\right) \\
& C=\frac{1}{A_{11}^{*}}\left(b_{2}^{*}-\frac{c_{21}}{c_{11}} b_{1}^{*}-A_{12}^{*} D-A_{13}^{*} F\right)
\end{aligned}
$$

Here

$$
\begin{aligned}
& a_{11}=-\sinh \alpha h \quad a_{12}=-\cosh \alpha h \quad a_{13}=2\left(1-\mu_{1}\right) \cosh \alpha h-\alpha h \sinh \alpha h \\
& a_{14}=2\left(1-\mu_{1}\right) \sinh \alpha h-\alpha h \cosh \alpha h \quad a_{15}=0 \quad a_{21}=-\cosh \alpha h \\
& a_{22}=-\sinh \alpha h \quad a_{23}=\left(1-2 \mu_{1}\right) \sinh \alpha h-\alpha h \cosh \alpha h \\
& a_{24}=\left(1-2 \mu_{1}\right) \cosh \alpha h-\alpha h \sinh \alpha h \quad a_{25}=0 \quad a_{31}=-1 \quad a_{32}=1 \\
& a_{33}=0 \quad a_{34}=3-4 \mu_{1} \quad a_{35}=-\left(3-4 \mu_{1}\right) \quad a_{41}=-G_{1} \quad a_{42}=1 \\
& a_{43}=0 \quad a_{44}=G_{1}\left(1-2 \mu_{1}\right) \quad a_{45}=-G_{2}\left(1-2 \mu_{2}\right) \quad a_{51}=0 \\
& a_{52}=1-2 G_{1}\left(1-\mu_{1}\right) \quad a_{53}=2 G_{1}\left(1-\mu_{1}\right) \quad a_{54}=0 \\
& a_{55}=-2 G_{2}\left(1-2 \mu_{2}\right) \quad B_{1}=-\frac{P}{2 \pi G_{1} \alpha^{2}} \quad B_{2}=0 \\
& B_{3}=0 \quad B_{4}=0 \quad B_{5}=0 \\
& c_{11}=a_{22}-a_{12} \frac{a_{21}}{a_{11}} \quad c_{12}=a_{23}-a_{13} \frac{a_{21}}{a_{11}} \quad c_{13}=a_{24}-a_{14} \frac{a_{21}}{a_{11}} \\
& c_{14}=a_{25}-a_{15} \frac{a_{21}}{a_{11}} \quad c_{21}=a_{32}-a_{12} \frac{a_{31}}{a_{11}} \quad c_{22}=a_{33}-a_{13} \frac{a_{31}}{a_{11}} \\
& c_{23}=a_{34}-a_{14} \frac{a_{31}}{a_{11}} \quad c_{24}=a_{35}-a_{15} \frac{a_{31}}{a_{11}} \quad c_{31}=a_{42}-a_{12} \frac{a_{41}}{a_{11}} \\
& c_{32}=a_{43}-a_{13} \frac{a_{41}}{a_{11}} \quad c_{33}=a_{44}-a_{14} \frac{a_{41}}{a_{11}} \quad c_{34}=a_{45}-a_{15} \frac{a_{41}}{a_{11}} \\
& c_{41}=a_{52}-a_{12} \frac{a_{51}}{a_{11}} \quad c_{42}=a_{53}-a_{13} \frac{a_{51}}{a_{11}} \quad c_{43}=a_{54}-a_{14} \frac{a_{51}}{a_{11}} \\
& c_{44}=a_{55}-a_{15} \frac{a_{51}}{a_{11}} \quad A_{11}^{*}=c_{22}-c_{12} \frac{c_{21}}{c_{11}} \quad A_{12}^{*}=c_{23}-c_{13} \frac{c_{21}}{c_{11}}
\end{aligned}
$$




$$
\begin{aligned}
& A_{13}^{*}=c_{24}-c_{14} \frac{c_{21}}{c_{11}} \quad A_{21}^{*}=c_{32}-c_{12} \frac{c_{31}}{c_{11}} \quad A_{22}^{*}=c_{33}-c_{13} \frac{c_{31}}{c_{11}} \\
& A_{23}^{*}=c_{34}-c_{14} \frac{c_{31}}{c_{11}} \quad A_{31}^{*}=c_{42}-c_{12} \frac{c_{41}}{c_{11}} \quad A_{32}^{*}=c_{43}-c_{13} \frac{c_{41}}{c_{11}} \\
& A_{33}^{*}=c_{44}-c_{14} \frac{c_{41}}{c_{11}} \\
& b_{1}^{*}=-\frac{a_{21}}{a_{11}} B_{1} \quad b_{2}^{*}=-\frac{a_{31}}{a_{11}} B_{1} \quad b_{3}^{*}=-\frac{a_{41}}{a_{11}} B_{1} \quad b_{4}^{*}=-\frac{a_{51}}{a_{11}} B_{1} \\
& \Delta=\left(A_{22}^{*}-A_{12}^{*} \frac{A_{21}^{*}}{A_{11}^{*}}\right)\left(A_{33}^{*}-A_{13}^{*} \frac{A_{31}^{*}}{A_{11}^{*}}\right)-\left(A_{23}^{*}-A_{13}^{*} \frac{A_{21}^{*}}{A_{11}^{*}}\right)\left(A_{32}^{*}-A_{12}^{*} \frac{A_{31}^{*}}{A_{11}^{*}}\right) \\
& \Delta_{1}=M_{1}\left(A_{33}^{*}-A_{13}^{*} \frac{A_{31}^{*}}{A_{11}^{*}}\right)-M_{2}\left(A_{23}^{*}-A_{13}^{*} \frac{A_{21}^{*}}{A_{11}^{*}}\right) \\
& \Delta_{2}=M_{2}\left(A_{22}^{*}-A_{12}^{*} \frac{A_{21}^{*}}{A_{11}^{*}}\right)-M_{1}\left(A_{32}^{*}-A_{12}^{*} \frac{A_{31}^{*}}{A_{11}^{*}}\right) \\
& M_{1}=b_{3}^{*}-\frac{c_{31}}{c_{11}} b_{1}^{*}-\left(b_{2}^{*}-\frac{c_{21}}{c_{11}} b_{1}^{*}\right) \frac{A_{21}^{*}}{A_{11}^{*}} \quad M_{2}=b_{4}^{*}-\frac{c_{41}}{c_{11}} b_{1}^{*}-\left(b_{2}^{*}-\frac{c_{21}}{c_{11}} b_{1}^{*}\right) \frac{A_{31}^{*}}{A_{11}^{*}}
\end{aligned}
$$

By means of formulae (3.1), (3.2), we find the stress components $\left|x_{1}\right| \leqslant \ell_{1}, y_{1}=0$ and $L_{k}$ $\left(\left|x_{k}\right| \leqslant \ell_{k}, y_{k}=0, k=1,2, \ldots, N\right)$.

The boundary conditions of the problem for the second stress-strain state take the form: - for $y=0$

$$
\sigma_{y}^{(1)}=0 \quad \tau_{x y}^{(1)}=0
$$

- for $y=-h$

$$
\sigma_{y}^{(1)}=0 \quad \tau_{x y}^{(1)}=0
$$

— for $y_{1}=0$

$$
\sigma_{y_{1}}-\mathrm{i} \tau_{x_{1} y_{1}}= \begin{cases}-\left(\sigma_{y_{1}}^{1}-\mathrm{i} \tau_{x_{1} y_{1}}^{1}\right) & \text { on the crack faces } \\ \sigma_{s}-\mathrm{i} \tau_{s}-\left(\sigma_{y_{1}}^{1}-\mathrm{i} \tau_{x_{1} y_{1}}^{1}\right) & \text { on the end zone faces of the crack }\end{cases}
$$

- for $y_{k}=0,\left|x_{k}\right| \leqslant \ell_{k}$

$$
\sigma_{y_{k}}-\mathrm{i} \tau_{x_{k} y_{k}}=\sigma_{s}-\mathrm{i} \tau_{s}-\left(\sigma_{y_{k}}^{1}-\mathrm{i} \tau_{x_{k} y_{k}}^{1}\right) \quad(k=1,2, \ldots, N)
$$

By means of the Kolosov-Muskhelishvili formulae (Muskhelishvili, 1977), we represent boundary conditions (3.5)-(3.7) in the form of the boundary value problem for finding the two analytic functions $\Phi(z)$ and $\Psi(z)$

$$
\begin{array}{ll}
y=0 & \Phi(z)+\overline{\Phi(z)}+z \overline{\Phi^{\prime}(z)}+\overline{\Psi(z)}=0 \\
y=-h & \Phi(z)+\overline{\Phi(z)}+z \overline{\Phi^{\prime}(z)}+\overline{\Psi(z)}=0 \\
y_{k}=0 & \Phi\left(x_{k}\right)+\overline{\Phi\left(x_{k}\right)}+x_{k} \overline{\Phi^{\prime}\left(x_{k}\right)}+\overline{\Psi\left(x_{k}\right)}=F_{k}
\end{array}
$$

where

$$
\begin{aligned}
& F_{1}= \begin{cases}-\left(\sigma_{y_{1}}^{1}-\mathrm{i} \tau_{x_{1} y_{1}}^{1}\right) & \text { on the crack faces } \\
\sigma_{S}-\mathrm{i} \tau_{S}-\left(\sigma_{y_{1}}^{1}-\mathrm{i} \tau_{x_{1} y_{1}}^{1}\right) & \text { on the end zone faces of the crack }\end{cases} \\
& F_{k}=\sigma_{S}-\mathrm{i} \tau_{S}-\left(\sigma_{y_{k}}^{1}-\mathrm{i} \tau_{x_{k} y_{k}}^{1}\right)
\end{aligned}
$$


We look for the complex potentials $\Phi(z)$ and $\Psi(z)$ (Panasyuk et al., 1977) in the form

$$
\begin{aligned}
& \Phi(z)=\frac{1}{2 \pi} \sum_{k=0}^{N+1} \int_{-\ell_{k}}^{\ell_{k}} \frac{g_{k}(t)}{t-z_{k}} d t \\
& \Psi(z)=\frac{1}{2 \pi} \sum_{k=0}^{N+1} \mathrm{e}^{-2 \mathrm{i} \alpha_{k}} \int_{-\ell_{k}}^{\ell_{k}}\left[\frac{\overline{g_{k}(t)}}{t-z_{k}}-\frac{\overline{T_{k}} \mathrm{e}^{\mathrm{i} \alpha_{k}}}{\left(t-z_{k}\right)^{2}} g_{k}(t)\right] d t
\end{aligned}
$$

where

$$
T_{k}=t \mathrm{e}^{\mathrm{i} \alpha_{k}}+z_{k}^{0} \quad z_{k}=\mathrm{e}^{-\mathrm{i} \alpha_{k}}\left(z-z_{k}^{0}\right)
$$

Using transformation formulae (Muskhelishvili, 1977) in transfoming into the new system of coordinates

$$
\begin{aligned}
& \Phi_{k}\left(z_{k}\right)=\Phi\left(z_{k} \mathrm{e}^{\mathrm{i} \alpha_{k}}+z_{k}^{0}\right) \\
& \Psi_{k}\left(z_{k}\right)=\mathrm{e}^{2 \mathrm{i} \alpha_{k}}\left[\Psi\left(z_{k} \mathrm{e}^{\mathrm{i} \alpha_{k}}+z_{k}^{0}\right)+\bar{z}_{k}^{0} \Phi^{\prime}\left(z_{k} \mathrm{e}^{\mathrm{i} \alpha_{k}}+z_{k}^{0}\right)\right]
\end{aligned}
$$

we write complex potentials $\Phi_{n}\left(z_{n}\right)$ and $\Psi_{n}\left(z_{n}\right)$ for the considered problem in the system of coordinates $x_{n} O_{n} y_{n}$

$$
\begin{aligned}
& \Phi_{n}\left(z_{n}\right)=\frac{1}{2 \pi} \sum_{k=0}^{N+1} \int_{-\ell_{k}}^{\ell_{k}} \frac{g_{k}(t)}{t-z_{k}} d t \\
& \Psi_{n}\left(z_{n}\right)=\frac{1}{2 \pi} \sum_{k=0}^{N+1} \mathrm{e}^{2 \mathrm{i} \alpha_{n k}} \int_{-\ell_{k}}^{\ell_{k}}\left[\frac{\overline{g_{k}(t)}}{t-z_{k}}-\frac{\left(\overline{T_{k}}-z_{n}\right) \mathrm{e}^{\mathrm{i} \alpha_{k}}}{\left(t-z_{k}\right)^{2}} g_{k}^{0}(t)\right] d t
\end{aligned}
$$

where

$$
z_{k}=\mathrm{e}^{-\mathrm{i} \alpha_{k}}\left(z_{n} \mathrm{e}^{\mathrm{i} \alpha_{n}}+z_{n}^{0}-z_{k}^{0}\right) \quad \alpha_{n k}=\alpha_{n}-\alpha_{k}
$$

Having defined by the Kolosov-Muskhelishvili formula (Muskhelishvili, 1977) the stresses on the axis $x_{n}$ and substituting into boundary condition (3.8), after some transformations, we get a system of $N+2$ integral equations:

— for $|x|<\infty$

$$
\begin{gathered}
\int_{-\infty}^{\infty}\left[\frac{g_{0}^{0}(t)}{t-x}+g_{N+1}^{0}(t) K_{0, N+1}(t-x)+\overline{g_{N+1}^{0}(t)} L_{0, N+1}(t-x)\right] d t \\
=-\sum_{k=1}^{N} \int_{-\ell_{k}}^{\ell_{k}}\left[g_{k}^{0}(t) K_{0, k}(t, x)+\overline{g_{k}^{0}(t)} L_{0, k}(t, x)\right] d t \\
\int_{-\infty}^{\infty}\left[\frac{g_{N+1}^{0}(t)}{t-x}+g_{0}^{0}(t) K_{N+1,0}(t-x)+\overline{g_{0}^{0}(t)} L_{N+1,0}(t-x)\right] d t \\
=-\sum_{k=1}^{N} \int_{-\ell_{k}}^{\ell_{k}}\left[g_{k}^{0}(t) K_{N+1, k}(t, x)+\overline{g_{k}^{0}(t)} L_{N+1, k}(t, x)\right] d t
\end{gathered}
$$


— for $|x|<\ell_{n}(n=1,2 \ldots, N)$

$$
\begin{aligned}
& \int_{-\ell_{k}}^{\ell_{k}} \frac{g_{n}^{0}(t)}{t-x}+\sum_{k \neq n}\left[\int_{-\ell_{k}}^{\ell_{k}} g_{k}^{0}(t) K_{n k}(t, x)+\overline{g_{k}^{0}(t)} L_{n k}(t, x)\right] d t \\
& \quad+\int_{-\infty}^{\infty}\left[g_{0}^{0}(t) K_{n, 0}(t, x)+\overline{g_{0}^{0}(t)} L_{n, 0}(t, x)\right] d t \\
& \quad+\int_{-\infty}^{\infty}\left[g_{N+1}^{0}(t) K_{n, N+1}(t, x)+\overline{g_{N+1}^{0}(t)} L_{n, N+1}(t, x)\right] d t=\pi F_{n}(x)
\end{aligned}
$$

Here

$$
\begin{aligned}
& K_{0, N+1}(x)=K_{N+1,0}(x)=\frac{x}{x^{2}+h^{2}} \quad L_{0, N+1}(x)=\overline{L_{N+1,0}(x)}=\frac{\mathrm{i} h}{(x+\mathrm{i} h)^{2}} \\
& K_{0, k}(t, x)=\frac{\mathrm{e}^{\mathrm{i} \alpha_{k}}}{2}\left(\frac{1}{T_{k}-x-\mathrm{i} h / 2}+\frac{1}{\bar{T}_{k}-x+\mathrm{i} h / 2}\right) \\
& L_{0, k}(t, x)=\frac{\mathrm{e}^{-\mathrm{i} \alpha_{k}}}{2} \frac{\bar{T}_{k}-T_{k}+\mathrm{i} h}{\left(\bar{T}_{k}-x+\mathrm{i} h / 2\right)^{2}} \\
& K_{n, 0}(t, x)=\frac{1}{2}\left(\frac{1}{t+\mathrm{i} h / 2-X_{n}}+\frac{\mathrm{e}^{-2 \mathrm{i} \alpha_{n}}}{t-\mathrm{i} h / 2-\bar{X}_{n}}\right) \\
& L_{n, 0}(t, x)=\frac{1}{2}\left(\frac{1}{t-\mathrm{i} h / 2-\bar{X}_{n}}-\frac{t+\mathrm{i} h / 2-X_{n}}{\left(t-\mathrm{i} h / 2-\bar{X}_{n}\right)^{2}} \mathrm{e}^{-2 \mathrm{i} \alpha_{n}}\right) \\
& K_{N+1, k}(t, x)=\frac{\mathrm{e}^{\mathrm{i} \alpha_{k}}}{2}\left(\frac{1}{T_{k}-x+\mathrm{i} h / 2}+\frac{1}{\bar{T}_{k}-x-\mathrm{i} h / 2}\right) \\
& L_{N+1, k}(t, x)=\frac{\mathrm{e}^{-\mathrm{i} \alpha_{k}}}{2} \frac{\bar{T}_{k}-T_{k}-\mathrm{i} h}{\left(\bar{T}_{k}-x-\mathrm{i} h / 2\right)^{2}} \\
& K_{n, N+1}(t, x)=\frac{1}{2}\left(\frac{1}{t-\mathrm{i} h / 2-X_{n}}+\frac{\mathrm{e}^{-2 \mathrm{i} \alpha_{n}}}{t+\mathrm{i} h / 2-\bar{X}_{n}}\right) \\
& L_{n, N+1}(t, x)=\frac{1}{2}\left(\frac{1}{t+\mathrm{i} h / 2-\bar{X}_{n}}-\frac{t-\mathrm{i} h / 2-X_{n}}{\left(t+\mathrm{i} h / 2-\bar{X}_{n}\right)^{2}} \mathrm{e}^{-2 \mathrm{i} \alpha_{n}}\right) \\
& K_{n k}(t, x)=\frac{\mathrm{e}^{\mathrm{i} \alpha_{k}}}{2}\left(\frac{1}{T_{k}-X_{n}}+\frac{\mathrm{e}^{-2 \mathrm{i} \alpha_{n}}}{\left(\bar{T}_{k}-\bar{X}_{n}\right)^{2}}\right) \\
& L_{n k}(t, x)=\frac{\mathrm{e}^{-\mathrm{i} \alpha_{k}}}{2}\left(\frac{1}{\bar{T}_{k}-\bar{X}_{n}}-\frac{T_{k}-X_{n}}{\left(\bar{T}_{k}-\bar{X}_{n}\right)^{2}} \mathrm{e}^{-2 \mathrm{i} \alpha_{n}}\right) \\
& X_{n}=x \mathrm{i} \alpha_{n}+z_{n}^{0}
\end{aligned}
$$

For convenience, in (3.12) and (3.13) and further, we omit the index in $x_{n}$. From the system of $N+2$ singular integral equations (3.12) and (3.13) we exclude two unknown functions $g_{0}^{0}(t)$ and $g_{N+1}^{0}(t)$.

We can write the solutions to equations (3.12) in the following way

$$
\begin{aligned}
g_{0}^{0}(t) & =\int_{-\infty}^{\infty}\left[D_{0}(t) W_{1}(x-t)+\overline{D_{0}(t)} W_{2}(x-t)+D_{N+1}(t) W_{3}(x-t)\right. \\
& \left.+\overline{D_{N+1}(t)} W_{4}(x-t)\right] d t
\end{aligned}
$$




$$
\begin{aligned}
& g_{N+1}^{0}(t)=\int_{-\infty}^{\infty}\left[D_{0}(t) \overline{W_{3}}(x-t)+\overline{D_{0}(t)} \overline{W_{4}}(x-t)+D_{N+1}(t) \overline{W_{1}}(x-t)\right. \\
& \left.\quad+\overline{D_{N+1}(t)} \overline{W_{2}}(x-t)\right] d t
\end{aligned}
$$

Here

$$
\begin{aligned}
& D_{0}(x)=-\frac{1}{\pi} \sum_{k=1}^{N} \int_{-\ell_{k}}^{\ell_{k}}\left[g_{k}^{0}(t) K_{0, k}(t, x)+\overline{g_{k}^{0}(t)} L_{0, k}(t, x)\right] d t \\
& D_{N+1}(x)=-\frac{1}{\pi} \sum_{k=1}^{N} \int_{-\ell_{k}}^{\ell_{k}}\left[g_{k}^{0}(t) K_{N+1, k}(t, x)+\overline{g_{k}^{0}(t)} L_{N+1, k}(t, x)\right] d t \\
& W_{1}(x)=\frac{1}{2}\left[M_{1}(x)+N_{1}(x)\right] \quad W_{2}(x)=\frac{1}{2}\left[M_{2}(x)+N_{2}(x)\right] \\
& W_{3}(x)=\frac{1}{2}\left[M_{2}(x)-N_{2}(x)\right] \quad W_{4}(x)=\frac{1}{2}\left[M_{1}(x)-N_{1}(x)\right] \\
& M_{1}(x)=-\frac{\mathrm{i}}{4 \pi} \int_{-\infty}^{\infty} \frac{\operatorname{sgn} s\left[\mathrm{e}^{h|s|}+h(|s|+s)\right]}{\sinh |s| h+|s| h} \mathrm{e}^{\mathrm{i} s x} d s \\
& M_{2}(x)=\frac{\mathrm{i}}{4 \pi} \int_{-\infty}^{\infty} \frac{\operatorname{sgn} s}{\sinh |s| h+|s| h} \mathrm{e}^{\mathrm{i} s x} d s \\
& N_{1}(x)=-\frac{\mathrm{i}}{4 \pi} \int_{-\infty}^{\infty} \frac{\operatorname{sgn} s\left\{\left[\mathrm{e}^{h|s|}-h(|s|+s)\right] \mathrm{e}^{\mathrm{i} s x}-(1+\mathrm{i} s x)+h s\right\}}{\sinh |s| h-|s| h} d s \\
& N_{2}(x)=-\frac{\mathrm{i}}{4 \pi} \int_{-\infty}^{\infty} \frac{\operatorname{sgn} s\left(\mathrm{e}^{\mathrm{i} s x}-1-\mathrm{i} s x\right)}{\sinh |s| h-|s| h} d s
\end{aligned}
$$

Now substituting into (3.15) the expressions for $D_{0}(x)$ and $D_{N+1}(x)$ from (3.16), we find

$$
\begin{aligned}
& g_{0}^{0}(x)=-\sum_{k=1}^{N} \int_{-\ell_{k}}^{\ell_{k}}\left[g_{k}^{0}(u) M_{0, k}(u, x)+\overline{g_{k}^{0}(u)} N_{0, k}(u, x)\right] d u \\
& g_{N+1}^{0}(x)=-\sum_{k=1}^{N} \int_{-\ell_{k}}^{\ell_{k}}\left[g_{k}^{0}(u) M_{N+1, k}(u, x)+\overline{g_{k}^{0}(u)} N_{N+1, k}(u, x)\right] d u
\end{aligned}
$$

Here

$$
\begin{aligned}
& M_{0, k}(u, x)=\frac{1}{\pi} \int_{-\infty}^{\infty}\left[W_{1}(x-t) K_{0, k}(u, t)+W_{2}(x-t) \overline{L_{0, k}(u, t)}\right. \\
& \left.+W_{3}(x-t) K_{N+1, k}(u, t)+W_{4}(x-t) \overline{L_{N+1, k}(u, t)}\right] d t \\
& N_{0, k}(u, x)=\frac{1}{\pi} \int_{-\infty}^{\infty}\left[W_{1}(x-t) L_{0, k}(u, t)+W_{2}(x-t) \overline{K_{0, k}(u, t)}\right. \\
& \left.\quad+W_{3}(x-t) L_{N+1, k}(u, t)+W_{4}(x-t) \overline{K_{N+1, k}(u, t)}\right] d t \\
& M_{N+1, k}(u, x)=\frac{1}{\pi} \int_{-\infty}^{\infty}\left[\overline{W_{3}(x-t)} K_{0, k}(u, t)+\overline{W_{4}(x-t)} \overline{L_{0, k}(u, t)}\right. \\
& \left.\quad+\overline{W_{1}(x-t)} K_{N+1, k}(u, t)+\overline{W_{2}(x-t)} \overline{L_{N+1, k}(u, t)}\right] d t
\end{aligned}
$$




$$
\begin{aligned}
& N_{N+1, k}(u, x)=\frac{1}{\pi} \int_{-\infty}^{\infty}\left[\overline{W_{3}(x-t)} L_{0, k}(u, t)+\overline{W_{4}(x-t)} \overline{K_{0, k}(u, t)}\right. \\
& \left.+\overline{W_{1}(x-t)} L_{N+1, k}(u, t)+\overline{W_{2}(x-t)} \overline{K_{N+1, k}(u, t)}\right] d t
\end{aligned}
$$

Now substituting these formulas into (3.13), after some transformations, we get a system of $N$ singular integral equations of the considered problem for $|x| \leqslant \ell_{n}(n=1,2, \ldots, N)$

$$
\int_{-\ell_{k}}^{\ell_{k}} \frac{g_{k}^{0}(t)}{t-x} d t+\sum_{k=1}^{N} \int_{-\ell_{k}}^{\ell_{k}}\left[g_{k}^{0}(t) R_{n k}(t, x)+\overline{g_{k}^{0}(t)} S_{n k}(t, x)\right] d t=\pi F_{n}\left(x_{n}\right)
$$

where

$$
R_{n k}(t, x)=\left(1-\delta_{n k}\right) K_{n k}(t, x)+r_{n k}(t, x) \quad S_{n k}(t, x)=\left(1-\delta_{n k}\right) L_{n k}(t, x)+s_{n k}(t, x)
$$

and

$$
\begin{aligned}
& r_{n k}(t, x)=\int_{-\infty}^{\infty}\left[K_{n, 0}(\tau, x) M_{0, k}(t, \tau)+L_{n, 0}(\tau, x) \overline{N_{0, k}(t, \tau)}\right. \\
& \left.+K_{n, N+1}(\tau, x) M_{N+1, k}(t, \tau)+L_{n, N+1}(\tau, x) \overline{N_{N+1, k}(t, \tau)}\right] d \tau \\
& s_{n k}(t, x)=\int_{-\infty}^{\infty}\left[K_{n, 0}(\tau, x) N_{0, k}(t, \tau)+L_{n, 0}(\tau, x) \overline{M_{0, k}(t, \tau)}\right. \\
& \left.\quad+K_{n, N+1}(\tau, x) N_{N+1, k}(t, \tau)+L_{n, N+1}(\tau, x) \overline{M_{N+1, k}(t, \tau)}\right] d \tau
\end{aligned}
$$

After substituting into (3.21) integrals (3.18), the kernels $r_{n k}(t, x)$ and $s_{n k}(t, x)$ will be represented by three-fold iterated integrals. After integration, these expressions may be represented by single integrals.

Omitting very bulky calculations, for the kernels $r_{n k}(t, x)$ and $s_{n k}(t, x)$ we finally find

$$
\begin{aligned}
& r_{n k}(t, x)=\int_{0}^{\infty}\left[\left(\frac{1}{\sinh h s+h s}+\frac{1}{\sinh h s-h s}\right) H_{n k}\left(X_{n}, T_{k}, s, \alpha_{n}, \alpha_{k}\right)\right. \\
& \left.+\left(\frac{1}{\sinh h s+h s}-\frac{1}{\sinh h s-h s}\right) G_{n k}\left(X_{n}, T_{k}, s, \alpha_{n}, \alpha_{k}\right)\right] d s \\
& s_{n k}(t, x)=\int_{0}^{\infty}\left[\left(\frac{1}{\sinh h s+h s}-\frac{1}{\sinh h s-h s}\right) H_{n k}\left(X_{n}, \bar{T}_{k}, s, \alpha_{n},-\alpha_{k}\right)\right. \\
& \left.+\left(\frac{1}{\sinh h s+h s}+\frac{1}{\sinh h s-h s}\right) G_{n k}\left(X_{n}, \bar{T}_{k}, s, \alpha_{n},-\alpha_{k}\right)\right] d s
\end{aligned}
$$

where

$$
\begin{aligned}
& H_{n k}\left(X_{n}, \bar{T}_{k}, s, \alpha_{n},-\alpha_{k}\right)=\frac{\mathrm{e}^{\mathrm{i} \alpha_{k}}}{4}\left\{\sin \left(X_{n}-\bar{T}_{k}\right) s\right. \\
& \quad-\sin \left(T_{k}-\bar{X}_{n}\right) s\left\langle h s+\mathrm{e}^{-2 \mathrm{i} \alpha_{n}}\left[1-h s+s^{2}\left(T_{k}-\bar{T}_{k}\right)\left(\bar{X}_{n}-X_{n}\right)+h^{2} s^{2}\right]\right\rangle \\
& \quad+\left\langle s\left(T_{k}-\bar{T}_{k}\right)-\mathrm{e}^{-2 \mathrm{i} \alpha_{n}}\left[\left(T_{k}-\bar{T}_{k}\right)+h s^{2}\left(\bar{X}_{n}-X_{n}-T_{k}+\bar{T}_{k}\right)\right]\right\rangle \cos \left(T_{k}-\bar{X}_{n}\right) s \\
& \left.\quad+\mathrm{e}^{-h s}\left[\sin \left(T_{k}-X_{n}\right) s+\mathrm{e}^{-2 \mathrm{i} \alpha_{n}} \sin \left(\bar{T}_{k}-\bar{X}_{n}\right) s\right]\right\} \\
& G_{n k}\left(X_{n}, T_{k}, s, \alpha_{n}, \alpha_{k}\right)=\frac{\mathrm{e}^{\mathrm{i} \alpha_{k}}}{4}\left\{-\left[1+\mathrm{e}^{-2 \mathrm{i} \alpha_{n}}(-1+h s)\right] \sin \left(\bar{T}_{k}-\bar{X}_{n}\right) s-h s \sin \left(T_{k}-X_{n}\right) s\right. \\
& \quad-s\left(T_{k}-\bar{T}_{k}\right) \cos \left(T_{k}-X_{n}\right) s-\mathrm{e}^{-2 \mathrm{i} \alpha_{n}}\left(\bar{X}_{n}-X_{n}\right) s \cos \left(\bar{T}_{k}-\bar{X}_{n}\right) s+\mathrm{e}^{-h s}\left[\sin \left(T_{k}-\bar{X}_{n}\right) s\right. \\
& \left.\left.\quad-\mathrm{e}^{-2 \mathrm{i} \alpha_{n}} \sin \left(T_{k}-\bar{X}_{n}\right) s+\mathrm{e}^{-2 \mathrm{i} \alpha_{n}} \sin \left(\bar{X}_{n}-X_{n}-T_{k}+\bar{T}_{k}\right) s \cos \left(T_{k}-\bar{X}_{n}\right) s\right]\right\}
\end{aligned}
$$


Note that the functions $r_{n k}(t, x)$ and $s_{n k}(t, x)$ are regular. They define the effect of faces of the band on the stress state near the crack tips. To the system of singular equations (3.19) for internal cracks, we should add the additional conditions

$$
\int_{-\ell_{k}}^{\ell_{k}} g_{k}^{0}(t) d t=0 \quad(k=1,2, \ldots, N)
$$

Using the procedure for converting (Panasyuk et al., 1977; Mirsalimov, 1987) at conditions (3.24), the system of complex singular integral equations (3.19) is reduced to a system of $N \times M$ algebraic equations for determining the $N \times M$ unknowns $g_{k}^{0}\left(t_{m}\right)(k=1,2, \ldots, N$; $m=1,2, \ldots, M)$

$$
\begin{aligned}
& \frac{1}{M} \sum_{m=1}^{M} \sum_{k=1}^{N} \ell_{k}\left[g_{k}^{0}\left(t_{m}\right) R_{n k}\left(\ell_{k} t_{m}, \ell_{n} x_{r}\right)+\overline{g_{k}^{0}\left(t_{m}\right)} S_{n k}\left(\ell_{k} t_{m}, \ell_{n} x_{r}\right)\right]=F_{n}^{0}\left(x_{r}^{0}\right) \\
& \sum_{m=1}^{M} g_{n}^{0}\left(t_{m}\right)=0 \quad(n=1,2, \ldots, N)
\end{aligned}
$$

If in (3.25) we pass to complex conjugate values, we get one more $N \times M$ algebraic equations.

For completeness of algebraic equations, we need $2 \times N$ complex equations determining the sizes of prefracture zones.

The solution of the system of integral equations is sought in the class of everywhere bounded functions (stresses). Consequently, it is necessary to add to system (3.25) the conditions of stress boundedness at the ends of the crack and prefracture zones $x_{k}= \pm l_{k}(k=1,2, \ldots, N)$. These conditions are of the form

$$
\begin{aligned}
& \sum_{m=1}^{M}(-1)^{m} g_{n}^{0}\left(t_{m}\right) \cot \frac{2 m-1}{4 M} \pi=0 \quad(n=1,2, \ldots, N) \\
& \sum_{m=1}^{M}(-1)^{M+m} g_{n}^{0}\left(t_{m}\right) \tan \frac{2 m-1}{4 M} \pi=0
\end{aligned}
$$

The obtained resolving systems of equations can be determines under the given external load the stress-strain state of the coating linked with elastic foundation in the availability of a crack and arbitrary number of prefracture zones in the coating. The united resolving system of equations becomes nonlinear because of the unknown values $l_{k}(k=1,2, \ldots, N)$. For its solution, we use the method of successive approximations the essence of which is the following. We solve system (3.25) at some definite values $l_{k}^{*}(k=1,2, \ldots, N)$ of the sizes of prefracture zones and the crack end zones with respect to the remaining unknowns. The remaining unknowns enter into the system linearly. The values of $l_{k}^{*}$ and the found quantities $g_{k}\left(t_{m}\right)$ are substituted into (3.26), i.e. into the unused equations of the system. The taken values of the parameters $l_{k}^{*}$ and the appropriate values $g_{k}\left(t_{m}\right)$ will not, generally speaking, satisfy equations (3.26). Therefore, by selecting the values of the parameters $l_{k}^{*}$, we will repeat the calculations until equations (3.26) of system (3.25) and (3.26) are satisfied with the given accuracy. At each approximation, the algebraic system is solved by the Gauss method with choosing the principal element.

Using the solution of the problem, calculate the opening on the faces of the crack and prefracture zones

$$
-\frac{1+\kappa}{2 G_{1}} \frac{\pi \ell_{k}}{M} \sum_{m=1}^{M_{1}} g_{k}\left(t_{m}\right)=v_{k}\left(x_{0 k}, 0\right)-\mathrm{i} u_{k}\left(x_{o k}, 0\right) \quad(k=1,2, \ldots, N)
$$

Here, $M_{1 k}$ is the number of nodal points contained in the interval $\left(-l_{k}, x_{0 k}\right)$. 
For the displacement vector modulus on the faces of the crack and prefracture zone for $x=x_{0 k}$, we have

$$
V_{0 k}=\sqrt{u_{k}^{2}+v_{k}^{2}}=\frac{1+\kappa}{2 G_{1}} \frac{\pi \ell_{k}}{M} \sqrt{A_{k}^{2}+B_{k}^{2}}
$$

where

$$
A_{k}=\sum_{m=1}^{M_{1 k}} v_{k}\left(t_{m}\right) \quad B_{k}=\sum_{m=1}^{M_{1} k} u_{k}\left(t_{m}\right) \quad(k=1,2, \ldots, N)
$$

To determine the external load at which the crack propagation occurs, we use the criterium of critical opening of crack faces at the foundation of the plastic deformations zone. Then the condition determining the limiting value of the external load will be the equality

$$
V_{01}\left(\lambda_{11}\right)=\delta_{c} \quad V_{01}\left(\lambda_{21}\right)=\delta_{c}
$$

where $\delta_{c}$ is a characteristic of the fracture toughness of the coating material defined experimentally.

The obtained solution of the problems allows one to predict the appearance of new cracks in the coating material. Tp achieve that, the problem statement should be complemented with the condition (criterion) of the crack appearance (discontinuity of interparticle bonds of the material). In place of such a condition, we accept the criterium of critical opening of prefracture zone faces

$$
\left|\left(v_{k}^{+}-v_{k}^{-}\right)-\mathrm{i}\left(u_{k}^{+}-u_{k}^{-}\right)\right|=\delta_{c r} \quad(k=2, \ldots, N)
$$

where $\delta_{c r}$ is the characteristics of resistance of the material to cracking.

Using the obtained solution, we can write the limit condition in the form

$$
V_{0 k}\left(x_{k}^{*}\right)=\delta_{c r} \quad(k=2, \ldots, N)
$$

where $x_{k}^{*}$ is the coordinate of the point of the prefracture zone at which discontinuity of the material interparticle bonds occurs.

These additional conditions enables finding the coating parameters at which new cracks appear in the coating cross section. Dependences of the length of the crack-tip zone $d_{1}=\left(l_{1}-\lambda_{11}\right) / l_{1}$ on the value of the load $p_{*}=P / h \sigma_{s}$ for different values of the crack length $l_{*}=\left(\lambda_{21}-\lambda_{11}\right) / l_{1}$ for $\alpha_{1}=45^{\circ}$ and $z_{0}=(0.05 h-\mathrm{i} 0.25 h)$ are depicted in Fig. 2 . The dependences of the prefracture zone length $l_{2} / h$ on the dimensionless value of the external load $P / h \sigma_{s}$ under different orientation angles of the prefracture zone location in the case $l_{*}=0.75$ are depicted in Fig. 3. The dependences of the opening of prefracture zone faces $\delta / \delta_{0}$ along the prefracture zone $x_{2} / l_{2}$ at different orientation angles of the prefracture zone location in the case $l_{*}=0.75$ are depicted in Fig. 4. Here $\delta_{0}=\pi E_{1} \delta_{c} / 8 \sigma_{s}$. Figure 5 represents the dependence of the critical load $p_{c}=P / h \sigma_{s}$ on the dimensionless length of the crack $\lambda=l_{*} / h$ for $\alpha_{1}=45^{\circ}$.

\section{Conclusions}

Experimental data from operational practice of the pair "coating-elastic foundation" convincingly show that at the design stage it is necessary to take into attention the cases when the coating may have damages and cracks. The existing methods of strength analysis of the pair "coating-elastic foundation" ignore this case. Such a situation makes it impossible to design a pair "coating-foundation" with minimal specific consumption of materials at guaranteed reliability and durability. 


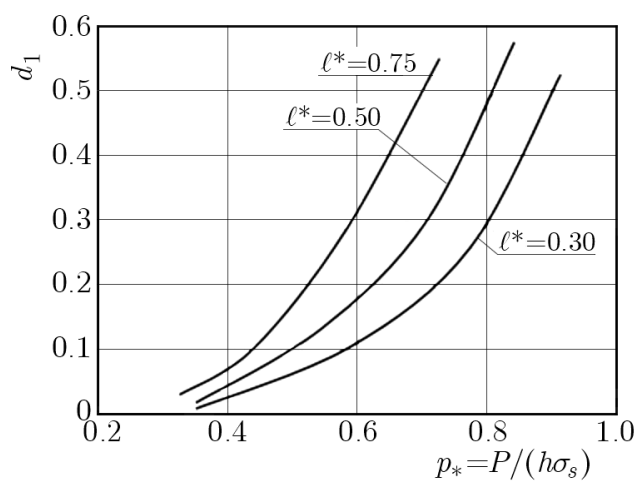

Fig. 2. Dependence of the length of the left end zone $d_{1}=\left(\ell_{1}-\lambda_{11}\right) / R_{1}$ on the dimensionless external load $p_{*}=P / h \sigma_{s}$ for different values of the crack length $\ell_{*}=\left(\lambda_{21}-\lambda_{11}\right) / \ell_{1}$ for $\alpha_{1}=45^{\circ}$ and $z_{0}=0.05 h-\mathrm{i} 0.25 h$

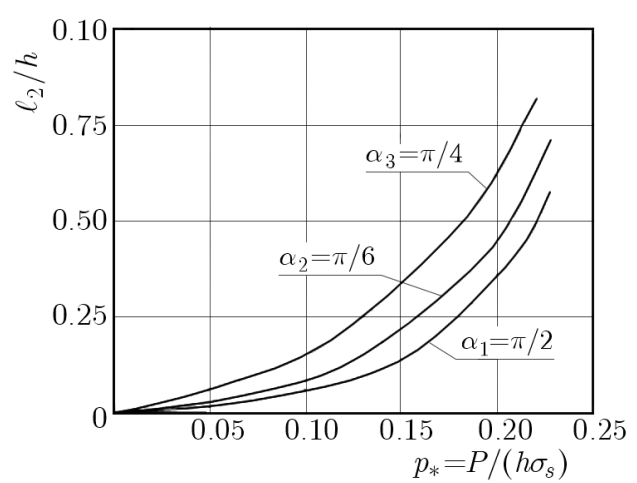

Fig. 3. Dependence of the prefracture zone length $\ell_{2} / h$ on the dimensionless length of the external load $P / h \sigma_{s}$ at different orientation angles of the prefracture zone location for the case $\ell_{*}=0.75$

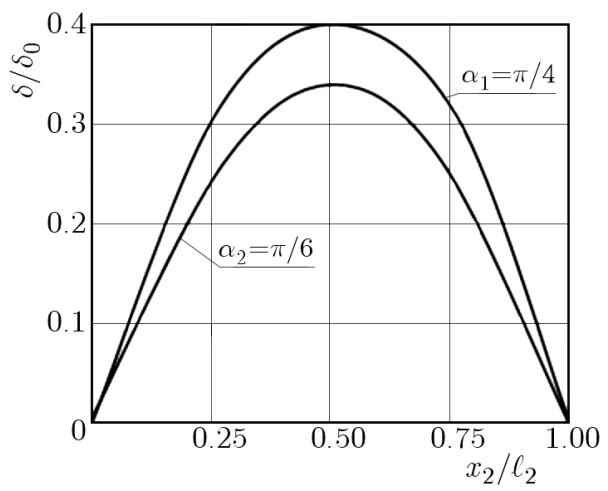

Fig. 4. Dependence of the prefracture zone faces opening $\delta / \delta_{0}$ along the prefracture zone at different orientation angles of the prefracture zone location for the case $x_{2} / \ell_{2}$. Here $\delta_{0}=\pi E_{1} \delta_{c} / 8 \sigma_{s}$

In this connection, it is necessary to realize the limit analysis of the pair "coating-foundation" in order to establish ultimate loads at which cracking and crack growth in the coating occurs. The size of the limiting minimal prefracture zone at which a crack appears should be considered as a design characteristic of the coating material.

Based on the suggested designed model that takes into account the availability of damages (zones of weakened interparticle bonds of the material) and cracks with end zones in the coating, we developed a method of calculation of coating parameters at which cracking and crack growth occurs. Knowing the basic values of critical parameters of cracking and the effect of materials properties on them, cracking and crack growth phenomenon may be controlled by means of design-technological decisions at the design stage. 


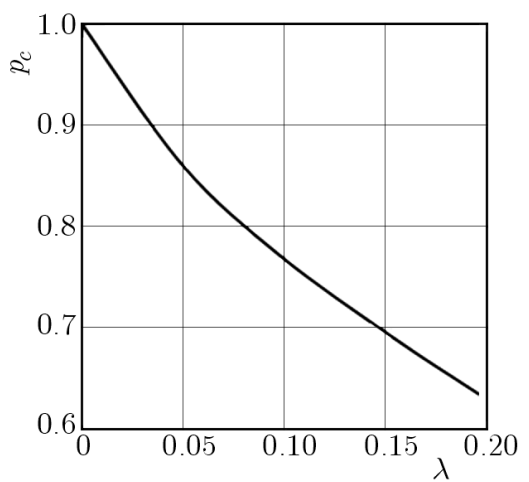

Fig. 5. Dependence of the critical load $p_{c}=P / h \sigma_{s}$ on the dimensionless length of the crack $\lambda=\ell_{1} / h$ for $\alpha_{1}=45^{\circ}$

Numerical realization of the obtained equations enables solution of the following practically important design problems:

1) to estimate the guaranteed resource of the pair "coating-elastic foundation" with regard to expected defects and loading conditions;

2) to establish the admissible deficiency level and maximum values of workload ensuring sufficient reliability reserve;

3) to choose materials with necessary complex of static and cyclic fracture toughness characteristics.

\section{References}

1. Ameri M., Mansourian A., Heidary Khavas M., Aliha M.R.M., Ayatollahi M.R., 2011, Cracked asphalt pavement under traffic loading - a 3D finite element analysis, Engineering Fracture Mechanics, 78, 8, 1817-1826

2. Dugdale D.S., 1960, Yielding of steel sheets containing slits, Journal of the Mechanics and Physics of Solids, 8, 100-108

3. HAJ-Ali R., 2009, Cohesive micromechanics: a new approach for progressive damage modeling in laminated composites, International Journal of Damage Mechanics, 18, 8, 691-720

4. Hasanov Sh.H., 2010, Calculated method of research of fatigue fracture of the road covering, Structural Mechanics of Engineering Constructions and Buildings, 2, 14-20

5. Hasanov Sh.H., 2013, Solution of fracture mechanics for the transverse crack in the cross section of road cover, Journal of Mechanics of Machines, Mechanisms and Materials, 2, 23, 35-40

6. Hasanov Sh.H., Mirsalimov V.M., 2014, Modeling of stress-strain state of road covering with cracks, Acta Polytechnica Hungarica, 11, 215-234

7. Leonov M.Ya., Panasyuk V.V., 1959, Propagation of fine cracks in solid body (in Russian), Prikladnaya Mekhanika, 5, 391-401

8. Kulchytsky-Zhyhailo R., Rogowski G., 2007, Stresses of hard coating under sliding contact, Journal of Theoretical and Applied Mechanics, 45, 4, 753-771

9. Mirsalimov V.M., Rustamov B.E., 2012, Effect of damages on crack-visible of the cavity opening displacement in burning solid fuel, International Journal of Damage Mechanics, 21, 373-384

10. Mirsalimov V.M., Rustamov B.E., 2012, Interaction of prefracture zones and crack-visible cavity in a burning solid with mixed boundary conditions, Acta Mechanica, 223, 627-643

11. Mirsalimov V.M., 1987, Non-One Dimensional Elastoplastic Problems (in Russian), Nauka, Moscow 
12. Muskhelishvili N.I., 1977, Some Basic Problem of Mathematical Theory of Elasticity, Kluwer, Amsterdam

13. Panasyuk V.V., 1991, Mechanics of Quasibrittle Fracture of Materials (in Russian), Naukova Dumka, Kiev

14. Panasyuk V.V, Savruk M.P., Datsyshyn A.P., 1977, A general method of solution of twodimensional problems in the theory of cracks, Engineering Fracture Mechanics, 9, 481-497

15. Rusinko A., Rusinko K., 2011, Plasticity and Creep of Metals, Springer, Berlin

16. Tukashev J.B., Adilhanova L.A., 2010, Investigation of stress-strain state of road covering, Geology, Geography and Global Energy, 37, 2, 163-165

17. Uflyand YA.S., 1967, Integral Transforms in the Theory of Elasticity (in Russian), Nauka, Leningrad

Manuscript received October 20, 2014; accepted for print April 6, 2015 